\title{
A SIMPLE NOETHERIAN RING NOT MORITA EQUIVALENT TO A DOMAIN
}

\author{
J. T. STAFFORD ${ }^{1}$
}

\begin{abstract}
An example of Zalesskii and Neroslavskii is used to produce an example of a simple ring that is not Morita equivalent to a domain.
\end{abstract}

In [5] an example is given of a simple Noetherian ring with divisors of zero but without nontrivial idempotents. In this note we show that this example also gives a negative answer to the important question of whether simple Noetherian rings are Morita equivalent to domains, thus answering [1, Question 1, p. 113].

The ring is constructed in the following way. Let $k$ be a field of characteristic two. Define $R_{1}=k(y)\left[X, X^{-1}\right]$ for indeterminates $y$ and $X$. Let $g$ be the $k(y)$-automorphism of $R_{1}$, defined by $g(X)=y X$ and let $R_{2}$ be the twisted group ring $R_{1}\langle g\rangle$; i.e. as an additive group, $R_{2}$ is isomorphic to the ordinary group ring but multiplication is defined by $r g=g r^{g}$ for $r \in R_{1}$. Let $h$ be the $k(y)$-automorphism of $R_{2}$ defined by $h(X)=X^{-1}$ and $h(g)=$ $g^{-1}$ and define $S$ to be the twisted group ring $R_{2}\langle h\rangle$. The ring $S$ was first constructed in [5], where the following was proved.

THEOREM 1. $S$ is a simple Noetherian ring, not a domain, such that the only idempotents of $S$ are 0 and 1.

In [5], $S$ was actually defined as a localisation of a group ring over $k$. However, the characterisation given here is more convenient as it provides an easy method of calculating the Krull dimension of $S$, written Kdim $S$ (for our purposes the following definition suffices. Given a prime ring $R$ then $\mathrm{Kdim} R$ $=1$ if $R$ is not Artinian but $R / I$ is an Artinian module for any essential one-sided ideal $I$ ).

THEOREM 2. Kdim $S=1$.

Proof. Clearly $R_{1}$ is hereditary. Since $g$ leaves no ideal of $R_{1}$ invariant, $R_{2}$ is hereditary by [3, Theorem 2.3]. Thus $\mathrm{Kdim} R_{2}=1$ (see for example [2, Theorem 1.3]). But $S$ is finitely generated as a left or right $R_{2}$-module. So $\mathrm{K} \operatorname{dim} S \leqslant 1$ and clearly we have equality.

Received by the editors May 12, 1977.

AMS (MOS) subject classifications (1970). Primary 16A12, 16A48.

'Supported by the British Science Research Council through a NATO Research Fellowship. 
This result enables us to use the results of [4] to show that $S$ has the properties described in the title.

THEOREM 3. $S$ is a simple Noetherian ring that is not Morita equivalent to a domain.

Proof. Suppose $S$ is Morita equivalent to a domain $A$. Then $\operatorname{Kdim} A=1$ by Theorem 2 . Let $P$ be the image of $S$ under the equivalence of the right module categories. Since $S$ is not a domain, $P$ is not isomorphic to a right ideal of $A$. Thus, with rk $P$ being the rank of the biggest free module that can be embedded in $P$, we have rk $P \geqslant 2=1+\mathrm{Kdim} A$. So by [4, Theorem 2.1], $P \cong Q \oplus A$ for some nonzero module $Q$. But then $S \cong I \oplus J$ for some nonzero right ideals $I$ and $J$ of $S$. This implies that $S$ has nontrivial idempotents, which contradicts Theorem 1.

Since $(1+h) S$ has a periodic projective resolution, $S$ has infinite global dimension. Thus it is still possible that any simple Noetherian ring of finite global dimension is Morita equivalent to a domain. Indeed, using [4], it is possible to show that a simple Noetherian ring $R$, with finite global dimension and $\mathrm{Kdim} R=1$, is Morita equivalent to a domain.

\section{REFERENCES}

1. J. Cozzens and C. Faith, Simple Noetherian rings, Cambridge Univ. Press, Cambridge, 1975.

2. D. Eisenbud and J. C. Robson, Modules over Dedekind prime rings, J. Algebra 16 (1970), 67-85.

3. A. Shamsuddin, A note on a class of simple Noetherian rings, J. London Math. Soc. (2) 15 (1977), 213-216.

4. J. T. Stafford, Stable structure of non-commutative Noetherian rings. II, J. Algebra (to appear).

5. A. E. Zalesskii and O. M. Neroslavskii, There exists a Noetherian ring with divisors of zero but without idempotents, Comm. Algebra 5 (3) (1977), 231-244. (Russian)

Department of Mathematics, Brandeis University, Waltham, Massachusetts 02154 